\title{
FLOW CONTROL IN A SUPERSONIC INLET MODEL BY ELECTRICAL DISCHARGE
}

\author{
S. Leonov ${ }^{1}$, D. Yarantsev ${ }^{1}$, and F. Falempin ${ }^{2}$ \\ ${ }^{1}$ Joint Institute for High Temperature \\ Russian Academy of Sciences \\ Moscow, Russia \\ ${ }^{2}$ MBDA-France \\ France
}

This paper reports the results of experimental work on supersonic Flow Control by Weakly-Ionized Plasma. Particular objective of this activity is to demonstrate the steering effect of near-surface electrical discharge on supersonic flow structure in a model two-dimensional (2D) aerodynamic configuration with three-shock compression ramp. The experiments were arranged in lab-scale facility PWT-50 of JIHT RAS at connected pipe flow pattern. At rather sophisticated character of plasmaflow interaction, such a technique can be considered as a feasible method for accurate control of shocks position and angle in supersonic inlet.

\section{INTRODUCTION}

In early 2000s, specific capabilities of near-surface electrical discharge were demonstrated to control supersonic flow structure in a variety of geometrical configurations [1-4] including a compression ramp. There was shown that generation of surface localized discharges in a high-speed flow makes possible substantial change of the structure and parameters of flowfield. One of possible fields for a practical interest is the plasma steering effect in high-speed inlet configuration $[5,6]$. Comparing with mechanical one, the method of high-speed flow control based on plasma deposition possesses, at least, two advantages: inertialess and flexibility (electronic control of magnitude and location of plasma impact). Numerous potential applications can be considered (still extemporized in the most cases) for this technique $[3,7]$.

The effectiveness of plasma method for flow control critically depends on spatial distribution of power deposition. The realization of required level of gas processing for predefined region is nontrivial technical problem for a high-

This is an Open Access article distributed under the terms of the Creative Commons Attribution-Noncommercial License 3.0, which permits unrestricted use, distribution, and reproduction in any noncommercial medium, provided the original work is properly cited. 
speed flow. Plasma of electrical discharge appears in more or less homogeneous form at relatively low gas pressure $[1,4]$ limiting the field of application. At the same time the effectiveness of nonuniform plasma is demonstrated at higher gas density $[5,6]$, which is typical for practical aerodynamics. The effect of artificial flow separation behind discharge zone over a plane wall was found out and described in details at the first time [6].

The next important feature of electrical discharge application is that the gas heating occurs not only at the place of electric current location but also downstream of this region due to recombination and vibrational-translational (V-T) relaxation of both. Under optimal conditions, up to $90 \%$ of power deposition can be conserved in vibrational reservoir and dissociation of molecular gas. From the other side, this energy can be deposited later to provide more preferential profile of extrusive layer. The idea of using plasma with strong spatial nonuniformity, nonequilibrium composition, and unsteady temporal behavior gives chance to get a notable effect at technically reasonable level of power release. It was shown experimentally that the discharge in air demonstrates some advantage for shocks position control in duct-driven supersonic flow $[5,6]$.

This work examines several explicit aspects of the problem of discharge effect on supersonic flow pattern, namely: control of flow parameters in inlet's configuration; steering of the shocks angle and position; reduction of pressure losses; etc. For the better understanding of the problem, the experimental findings are compared with the results of numerical simulation [8].

\section{EXPERIMENTAL APPROACH}

Major objective of this work is to demonstrate the steering effect of plasma on supersonic flow structure in a compression ramp model. The configuration is close to the geometry of a simple 2D inlet of a supersonic air-breathing engine. Surface plasma generation near the inlet wedge is shown in Fig. 1 to shift the shock wave upstream. The angle of a new shock and the shock's position depend on the discharge configuration and parameters (the power release has the most important relevance but not only). This part of the work was devoted specifically to study the electrical discharge interaction with supersonic flow for model geometrical configuration in connected pipe scheme.

The test was performed in facility PWT-50 of JIHT RAS [5, 6, 8]. Incoming flow in duct with initial height $Y_{0}=60 \mathrm{~mm}$ and width $Z_{0}=72 \mathrm{~mm}$ has initial Mach number $\mathrm{M}=2$ and 2.5 and static air pressure $P_{\text {st }}=100-300$ Torr. In the section of interaction, the Mach number was a bit less: $\mathrm{M}=1.87$ and 2.35, approximately. The Reynolds number $\operatorname{Re}=(4 \div 10) \cdot 10^{6} \mathrm{~L}$, the boundary layer thickness in the testing zone $\delta=1.3-1.5 \mathrm{~mm}$, typical gas flow rate through the channel $G=0.6-1 \mathrm{~kg} / \mathrm{s}$, the duration of quasi-stationary phase $0.2-0.5 \mathrm{~s}$, and the total enthalpy flow rate $H \approx 200 \mathrm{~kW}$. Geometry of the model test is shown 


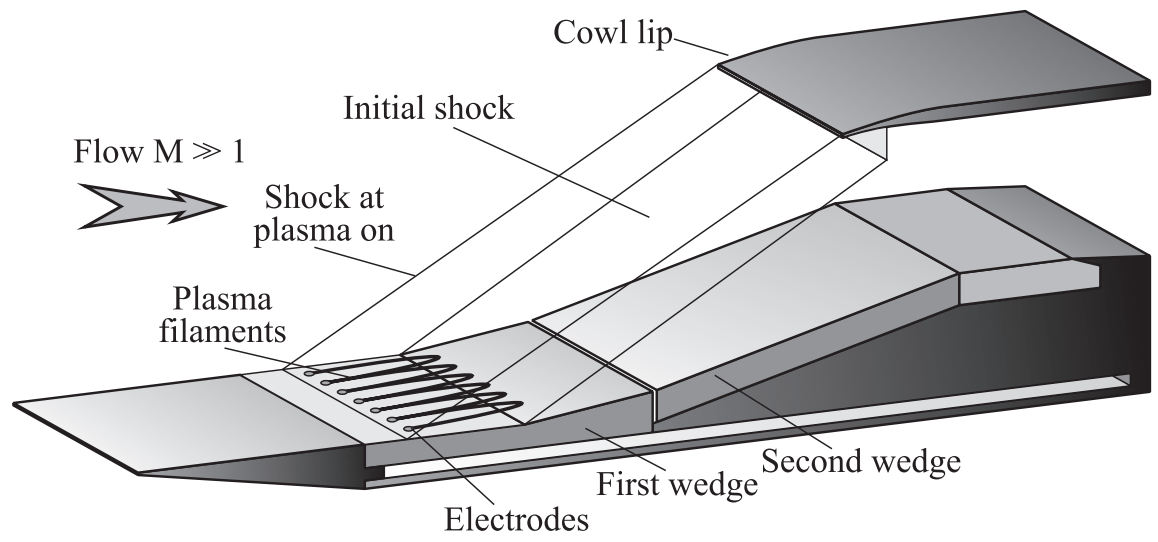

Figure 1 Draft scheme of experiment for demonstration of plasma steering effect in inlet. The second shock is not shown here

in Fig. 2. The model consists of two consequent planar wedges $\alpha_{1}=7^{\circ}, \alpha_{2}=14^{\circ}$ and divergent part. The opposite wall contains the backward wedge $\alpha_{3}=14^{\circ}$ to avoid the channel blockage. The electrodes' row includes 7 cooper tabs arranged by sequence cathode-anode-...-cathode and locates in $X=-18 \mathrm{~mm}$ upstream the first wedge. All electrodes were flush-mounted and did not affect the flow themselves.

Measuring system includes the following components: system sensors (pressure and temperature), pressure transducers (16 channels pressure distribution measurements), optical sensors, fast videocameras, electrical parameters sensors, Schlieren system, and Schlieren-streak system. Two methods give the main data on the flow structure and parameters: visualization and pressure measurements. In this experimental series, the measurements of plasma parameters were limited by power deposition, discharge resistivity, and discharge visualization. At the same time, the presence of the electrical discharge in the flow introduces extra difficulties in measurement of airflow parameters, particularly: electromagnetic noise, intensive illumination, unsteady heating, turbulization, nonequilibrium gas excitation, contaminations, etc. More detail information on measuring system can be found in [9]. Typical accuracy of pressure measurements was $d P_{\mathrm{st}}= \pm 2$ Torr and of averaged plasma power magnitude $d W_{\mathrm{pl}}= \pm 0.05 \mathrm{~kW}$. The characteristic time of pressure measurement was not worse than $0.3 \mathrm{~ms}$ for each channel; Schlieren system framerate was $f=2 \mathrm{kHz}$, and exposure time of each frame was $t=0.1 \mu \mathrm{s}$.

The measurements were focused on the following three issues:

(1) change of the shock wave structure of supersonic flow on compression ramp model; 


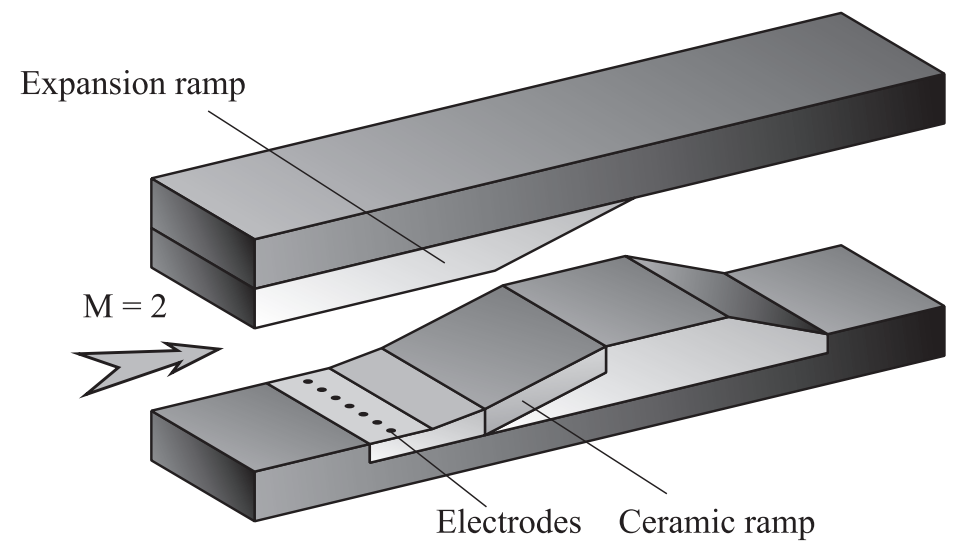

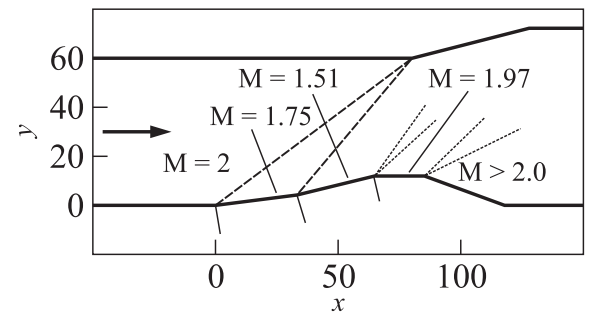

(a)

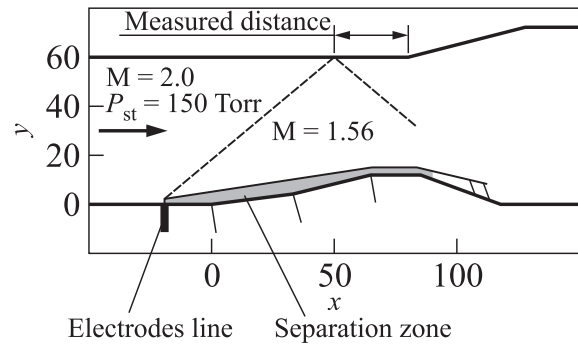

(b)

Figure 2 Geometry of the model for connected pipe test. Estimation of test result at initial flow velocity $\mathrm{M}=2$. Plasma off $(W=0)(a)$ and plasma on $(W=5-9 \mathrm{~kW})(b)$

(2) pressure redistribution in vicinity of ramp depending on plasma power; and

(3) the flow parameters modification behind the interaction area.

In accordance with these objectives, the electrical probing, Schlieren visualization, and pressure measurements were performed in each test. The test arrangement is presented in Fig. 3. Two separate optical windows were available for Schlieren observations as they are shown in Fig. 3: the first one illuminated flow structure in immediate vicinity of the interaction place, and the second window was located in downstream zone. The distance between them was about $x=50 \mathrm{~mm}$. Black points show the pressure tabs arrangement. Dashed lines reflect method of the Schlieren images processing: angle A0 = angle of Mach characteristics (it is a bit deviated from series to series); angle A1 (no plasma) or A3 (plasma on) = angle of the shock from the first wedge; angle A2 = angle from the second wedge; coordinate $X_{1}=$ the first shock position on the oppo- 


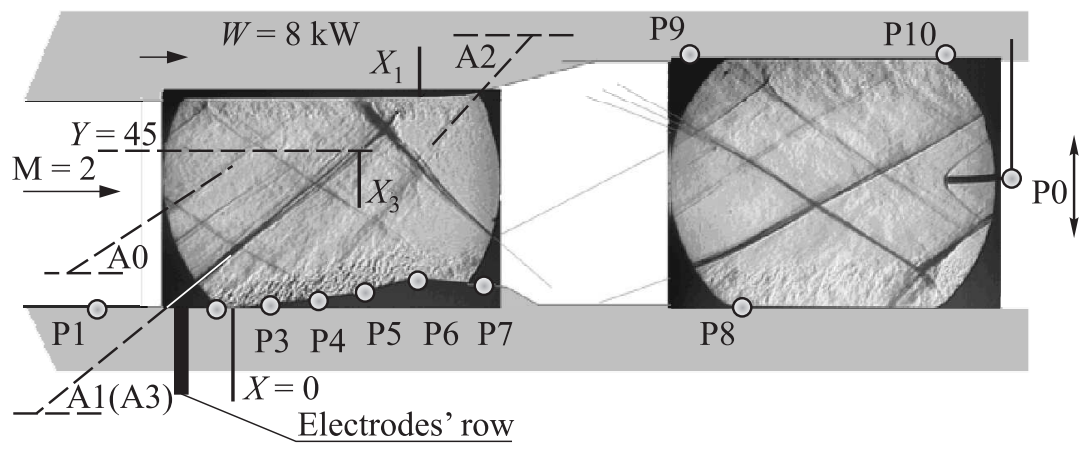

Figure 3 Experiments arrangement

site wall as it would be after extrapolation; $X_{3}=$ the first shock $x$-coordinate in section $Y=45 \mathrm{~mm}$. The shock angle A1 was measured as an average angle in a range from $Y=10$ to $45 \mathrm{~mm}$.

\section{DISCHARGE PARAMETERS}

The major properties of the near-surface quasi-direct-current electrical discharge were described in [6]. The discharge appears in the form of oscillating plasma filaments as it shown in Fig. 4a. Initial electrical breakdown occurs not far from the electrodes position. The individual filaments are blown down due to main

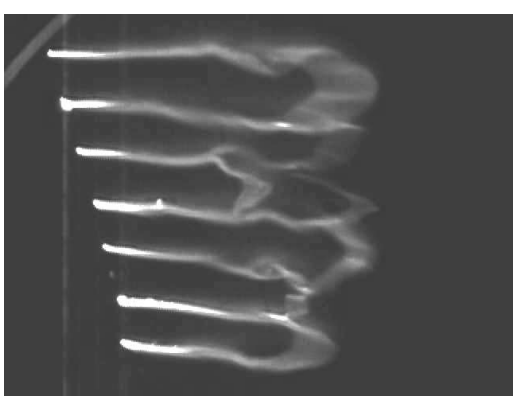

(a)

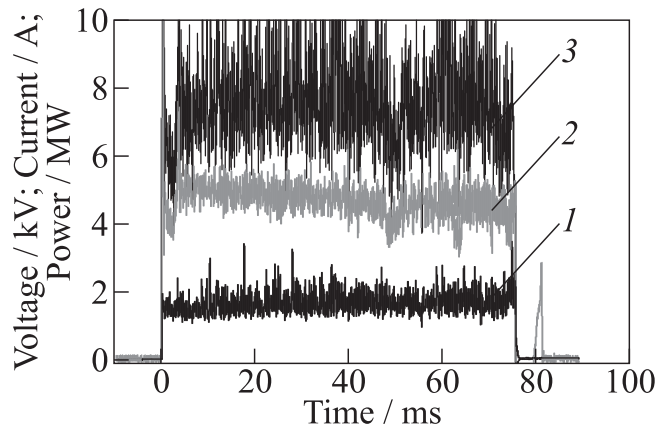

(b)

Figure 4 Discharge parameters: (a) instant photo, flow from left to right; and (b) typical voltage (1), current (2), and power $\left(W_{\mathrm{av}}=7.7 \mathrm{~kW}\right)(3)$ oscillograms (M $=1.84 ; P_{\mathrm{st}}=180$ Torr; $R_{a}=2 \mathrm{kOhm} ;$ and $\left.R_{c}=1 \mathrm{kOhm}\right)$ 
flow at velocity a bit less than the core value. The frequency of oscillations depends on flow speed, interelectrodes gap, and parameters of power supply. In the most cases, this value was $F=10-30 \mathrm{kHz}$ under the experimental conditions. The regulation of power release was performed by means of electrical current change in a range $W_{\mathrm{pl}}=3-17 \mathrm{~kW}$. The maximal translational gas temperature in discharge zone was measured by optical spectroscopy at about $T_{g} \approx 3000 \mathrm{~K}$; therefore, the V-T relaxation length was expected to be around several centimeters [5]. Typical oscillograms are presented in Fig. $4 b$.

\section{EXPERIMENTAL RESULTS}

Figures $5 a$ and $5 b$ present typical experimental data obtained for the model configuration mentioned above. Figure $5 a$ is the Schlieren image of undisturbed flow structure. Figure $5 b$ shows the flow structure when the plasma generator was switched on. An extrusive layer excited by plasma and the first shock moved upstream are well seen. There are three important statements that might be considered due to analysis of the Schlieren images:

(1) the first shock shifts upstream;

(2) the angle of the first shock decreases comparing with initial situation and depends on plasma power; and

(3) the second shock loses its intensity.

The generation of new shock wave structure starts from the first discharge breakdown and is followed by a cylindrical unsteady shock wave (SW in the picture) and thermal cavern formation. The initial stage is shown on instant

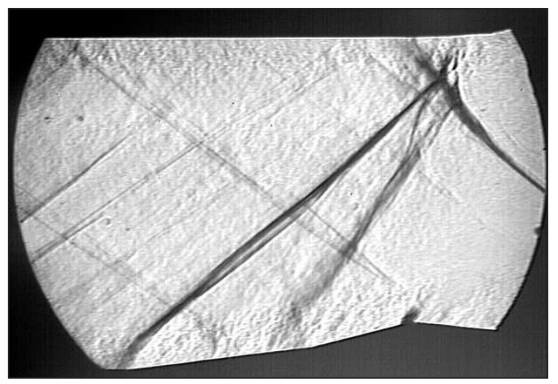

(a)

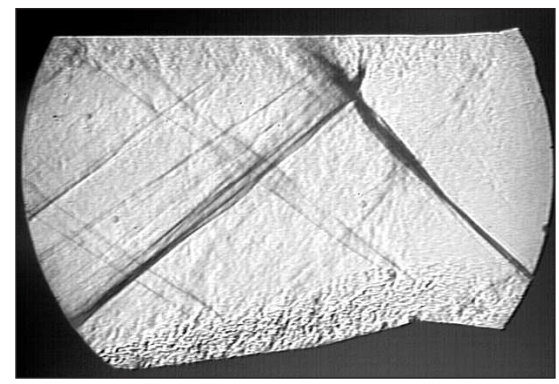

(b)

Figure 5 Schlieren picture for undisturbed flow $(a)$ and for plasma generation $W$ $=12 \mathrm{~kW}(b)$ 


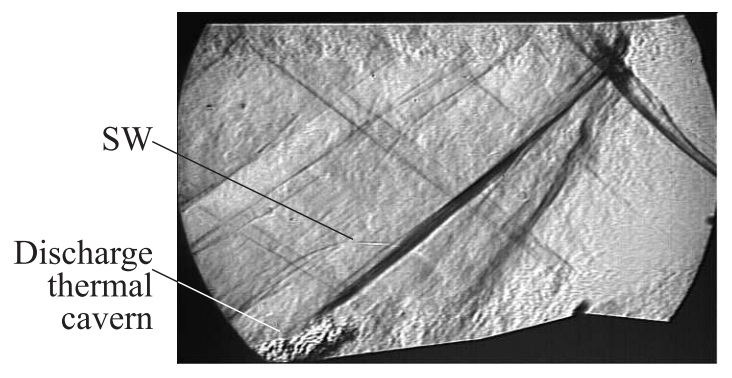

Figure 6 Schlieren image of initial stage of new shock structure formation. Delay $60 \mu \mathrm{s}$

Schlieren image in Fig. 6, which is recorded at $60 \mu$ s after the discharge initiation. Unfortunately, exact synchronization of Schlieren record and the discharge breakdown occurs rather difficult. To resolve the details of interaction at initial stage, an original Schlieren-streak technique was applied for visualization [9]. A main feature here is in application of the fast line-scan camera for Schlieren image acquisition along a predefined line of flowfield. In this particular case, the line of scanning was tuned in mid section of the channel $Y=30 \mathrm{~mm}$.

Two extra problems were under experimental analysis based on processing of Schlieren-streak records:

(1) duration of transitional mode (time for the new configuration establishment); and

(2) stability of plasma-induced shock (remember that the discharge is principally unstable).

A new shocks pattern is established in approximately $0.2 \mathrm{~ms}$ that is well visible in Schlieren-streak image (Fig. 7). The first shock is stabilized in a new position; the second shock looks to be disappeared in short time. A bit more time is needed for a new reflected shock formation. The longest time elapses for the restoration of initial structure after the discharge was switched off. The period of the discharge oscillation is about $50 \mu \mathrm{s}$, which is 5 times longer than the temporal resolution of the visualization technique, but which is less than the shock structure renovation. Therefore, the Schlieren-streak images prove the statement on stability of new shocks position.

The decrease of shock angle indicates that the conditions for the model flown around are significantly modified, namely, the pressure losses can be expected less than under initial configuration. The pressure measurements prove this premise as shown in Figs. 8 and 9. The integration of the pressure distribution along the model gives the reduction of tangential force in factor 0.7. Figure 9 presents the data on the total pressure measurements (Pitot tube pressure) across the 


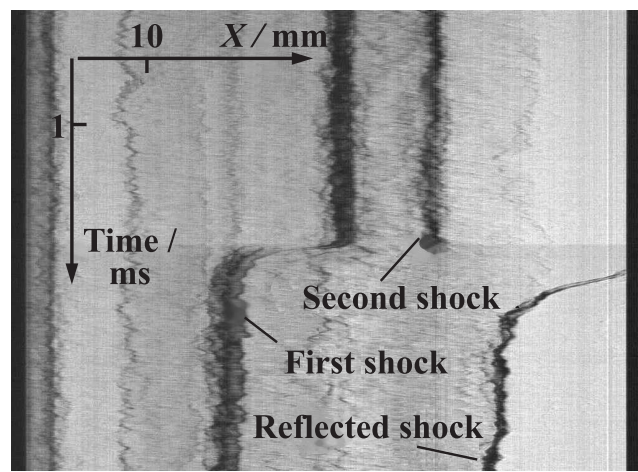

$68 \mathrm{~ms}$ are missed

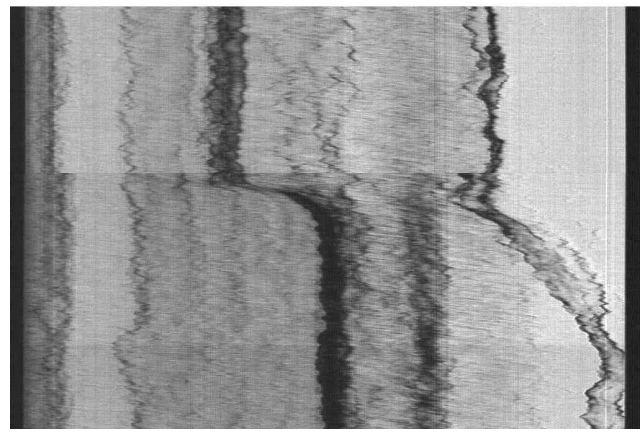

Figure 7 Schlieren-streak image, $Y=29 \mathrm{~mm}$

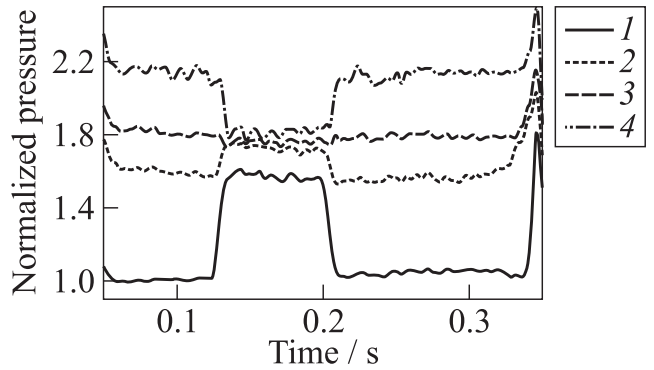

Figure 8 Static pressure redistribution on ramp under plasma generation, $W$ $=16.6 \mathrm{~kW}: 1-P_{2} / P_{1} ; 2-P_{3} / P_{1} ; 3-P_{4} / P_{1} ;$ and $4-P_{5} / P_{1}$ 


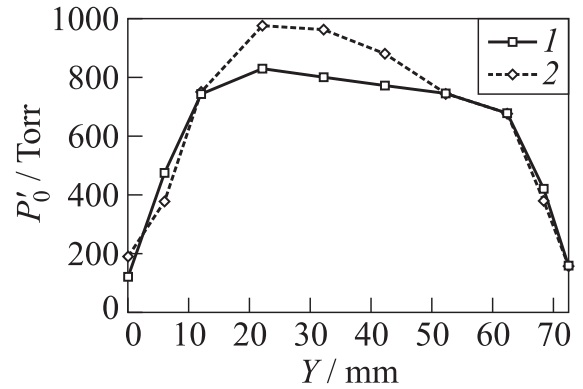

(a)

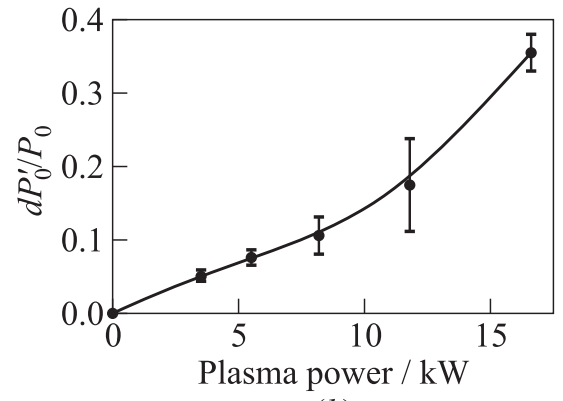

(b)

Figure 9 Plasma effect on total pressure downstream: $(a)$ tranversal pressure distribution, $X=210 \mathrm{~mm}$ ( 1 - no plasma and 2 - plasma on); and (b) total pressure vs. plasma power

channel downstream the model location. It can be considered the total pressure rise in the core of main flow, which reflects the reduction of the pressure losses in the model-associated shocks.

\section{THE FIRST SHOCK ANGLE AND POSITION VERSUS PLASMA POWER}

The Schlieren images allow recognizing the details of the flow structure (see Fig. 3). The first shock position $X_{3}$ was measured at $Y=45 \mathrm{~mm}$ (top wall has coordinate $Y=60 \mathrm{~mm}$ ) due to irregular shock reflection on the wall. The shock's position $X_{1}$ corresponds with the point of regular reflection. It is calculated based on $\mathrm{A} 1$ and $X_{3}$. Figure 10 presents the graph of the first shock angle depending on the plasma power release at two values of initial Mach number. The calculated results for $\mathrm{M}_{0}=2$ are shown here as well. Numerical modeling [8] of flow in experimental configuration was based on solution of three-dimensional (3D) time-dependent unstable Reynolds averaged Navier-Stocks equations (URANSmethod). The behavior is unstable at low level of plasma power: dashed line connects the experimental point here. The accuracy of angle measurement is not worse than $\pm 0.5^{\circ}$.

The graphs in Fig. 11 show the coordinates of shocks position at $Y=45 \mathrm{~mm}$ and on the top wall. The following statements can be written based on these results:

- zone of effective regulation of the shock position is covered by the power range $W_{\mathrm{pl}}=3-15 \mathrm{~kW}$. Following increase of the power can be useless. In dimensionless approach, it looks like follows: 


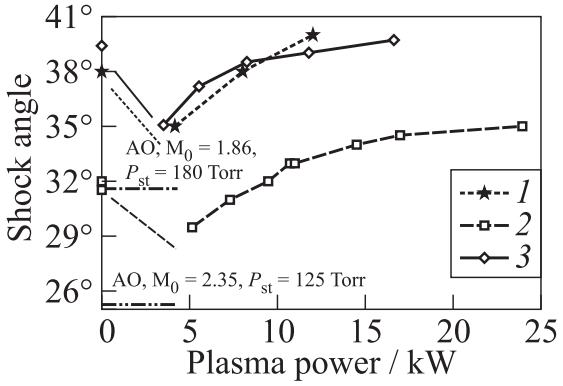

Figure 10 The first shock angle (1$\mathrm{M}_{0}=2$, and $\left.2-\mathrm{M}_{0}=2.35\right)$ vs. plasma power $\left(3-\right.$ plasma on, $\left.\mathrm{M}_{0}=1.86\right)$

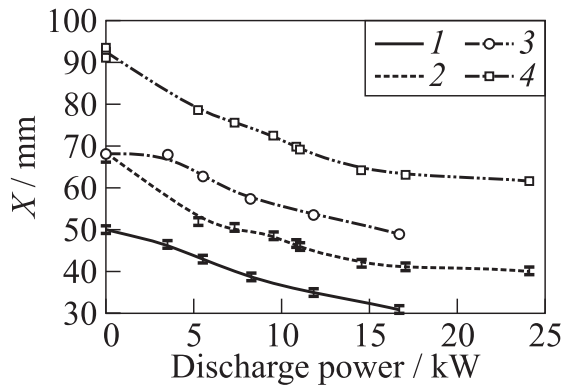

Figure 11 The first shock position at $Y=45 \mathrm{~mm}\left(1-\mathrm{M}_{0}=1.87 ;\right.$ and $2-$ $\left.\mathrm{M}_{0}=2.35\right) ;$ and on top wall $\left(3-\mathrm{M}_{0}\right.$ $=1.87 ;$ and $\left.4-\mathrm{M}_{0}=2.35\right)$ vs. plasma power

$$
w=\frac{W_{\mathrm{pl}}}{Z P_{\mathrm{st}}}=2-10[\mathrm{~kW} /(\mathrm{bar} \cdot \mathrm{cm})]
$$

where $w$ is the reduced plasma power; $Z$ is the channel width;

- in this experimental series, the range of regulation only a bit higher than the wedge-electrodes distance $X=-18 \mathrm{~mm}$; and

- in this experimental approach, the shock at $\mathrm{M}_{0}=2.35$ falls to the same position as for $\mathrm{M}_{0}=1.87$ at plasma power $W_{\mathrm{pl}}=10-12 \mathrm{~kW}$.

The data obtained show that the surface discharge burning upstream of the wedge leads to shifting of the root part of oblique shock upstream as well. At relatively small power release, the angle of the first shock is decreased significantly. The increase of the plasma power appeared in the rise of the first shock angle as it is presented in Fig. 10. The second shock occurs much weaker than under initial conditions. The power release below the value $W_{\mathrm{pl}}<3 \mathrm{~kW}$ causes an unstable behavior of the first shock. Such type of plasma-flow-model interaction can be called as "plasma screening" [6]. Downstream of this region, three main processes are in competition: cooling, turbulent mixing, and extra expansion due to energy relaxation from internal reservoir.

\section{CONCLUDING REMARKS}

The authors advocate a point of view that the plasma technology makes a sense for a correction of flow-field and support of high-speed flow regulation under the off-design operation modes. A theory [8] and the lab-scale experiments prove a 
hope on effective, inertialess, and adjustable control of the flow structure and gas parameters. In the most cases, this method is much more effective than mechanical one. In some cases, there are no other methods to achieve a required result.

The transversal electrical discharge affects the flow similar to a soft wedge, whose angle depends on the electrical power release. At exceeding the value about $W / P_{\mathrm{st}} \approx 10 z \mathrm{~W} /$ Torr, where $z$ is the discharge region depth, the flow separates [6] downstream with subsequent attachment or without it. The discharge generation is accompanied by the formation of the oblique shock. The amplitude, angle, and position of this shock wave can be regulated with the aid of electrical parameters variation. Specifically, the surface plasma generation near the inlet wedge is shown to regulate the angle of the first shock and to shift the shock wave in respect of the initial position.

In the case of air, a huge gradient postplasma extrusive layer is observed that is in accordance with an idea of the mechanism of slowed V-T relaxation. This layer "screens" the contoured model leading to some reduction of the pressure losses in the channel. The thickness and the length of this layer depend on static pressure of the gas and velocity of the flow. Despite of an unstable behavior of the discharge, the shocks' position was stable, predictable, and allowed regulation by means of discharge parameters. The experiments have demonstrated a promising ability of electrical discharge to improve the inlet's performance.

\section{ACKNOWLEDGMENTS}

Currently, this work is supported by MBDA-France. The authors appreciate Dr. A. Miller of MIPT for his help in numerical simulations.

\section{REFERENCES}

1. Merriman, S., E. Plonjes, and P. Palm. 2001. Shock wave control by nonequilibrium plasmas in cold supersonic gas flows. AIAA J. 39:1547-52.

2. Macheret, S., R. Miles, and M. Schneider. 2003. Comparative analysis of MHD and plasma methods of scramjet inlet control. AIAA Paper No. 2003-0170.

3. Bletzinger, P., B. N. Ganguly, D. VanWie, and A. Garscadden. 2005. Plasmas in high speed aerodynamics. Topical review. J. Phys. D: Appl. Phys. 38:R33-R57.

4. Shang, J. S., S. T. Surzhikov, R. Kimmel, et al. 2005. Mechanisms of plasma actuators for hypersonic flow control. Progr. Aerospace Sci. 41:642-68.

5. Leonov, S., V. Soloviev, and D. Yarantsev. 2006. High-speed inlet customization by surface electrical discharge. AIAA Paper No. 2006-0403. 
6. Leonov, S., and D. Yarantsev. 2008. Near-surface electrical discharge in supersonic airflow: Properties and flow control. J. Propul. Power 24(6):1168-81.

7. Moreau, E., C. Louste, G. Artana, M. Forte, and G. Touchard. 2006. Contribution of plasma control technology for aerodynamic applications. Plasma Proc. Polymers 3:697-707.

8. Leonov, S. B., A. A. Firsov, D. A. Yarantsev, F. Falempin, amd A. Miller. 2009. Flow control in model supersonic inlet by electrical discharge. AIAA Paper No. 2009-7367.

9. Leonov, S. B. 2010. Visualization of unsteady electrical discharges in high-speed flow. Keynote lecture. 10th ASV Conference (International) Proceedings. Nagar, Tamil Nadu, India. 1-11. 